\title{
BMJ Performance-based assessments and open demand for personal care in older Japanese people: a cross-sectional study
}

\author{
Hiroyuki Shimada, ${ }^{1}$ Takao Suzuki, ${ }^{2}$ Megumi Suzukawa, ${ }^{3}$ Hyuma Makizako, ${ }^{1}$ \\ Takehiko Doi, ${ }^{1}$ Daisuke Yoshida, ${ }^{1}$ Kota Tsutsumimoto, ${ }^{1}$ Yuya Anan, ${ }^{1}$ \\ Kazuki Uemura, ${ }^{1}$ Tadashi Ito, ${ }^{1}$ Sangyoon Lee, ${ }^{4}$ Hyuntae Park ${ }^{4}$
}

To cite: Shimada $\mathrm{H}$,

Suzuki T, Suzukawa M, et al. Performance-based assessments and demand for personal care in older Japanese people: a crosssectional study. BMJ Open 2013;3:e002424. doi:10.1136/bmjopen-2012002424

- Prepublication history for this paper are available online. To view these files please visit the journal online (http://dx.doi.org/10.1136/ bmjopen-2012-002424).

Received 30 November 2012 Revised 12 March 2013 Accepted 18 March 2013

This final article is available for use under the terms of the Creative Commons Attribution Non-Commercial 2.0 Licence; see http://bmjopen.bmj.com

For numbered affiliations see end of article.

Correspondence to Dr Hiroyuki Shimada; shimada@ncgg.go.jp

\section{ABSTRACT}

Objectives: To identify appropriate clinical tests for determining the demand for personal care in older Japanese people.

Design: Cross-sectional observation study.

Setting: Obu Study of Health Promotion for the Elderly (Obu, Aichi) and Tsukui Ordered Useful Care for Health (241 day-care centres) cohorts in Japan.

Participants: A total of 10351 individuals aged 65 years or older (6791 with personal care and 3560 without personal care) participated in the study.

Measures: Physical performance tests included grip strength, the chair stand test, walking speed at a comfortable pace, and the timed up-and-go test. Personal care was defined as participants who had been certified in the national social long-term care insurance in Japan.

Results: Individuals who received personal care showed a significantly poorer performance than those without personal care for all physical performance tests $(p<0.001)$. Gait speed was the most useful of the physical performance tests to determine the demand for personal care (receiver operating characteristic curve statistics: men, 0.92; women, 0.94; sensitivity: men, 86; women, 90; specificity: men, 85; women, 85). After adjustment for age, sex, cognitive impairment and other physical tests, all physical performance tests were individually associated with the demand for personal care. A slow gait speed $(<1 \mathrm{~m} / \mathrm{s})$ was more strongly correlated with the demand for personal care than other performance measures (gait speed OR: $5.9 ; 95 \% \mathrm{Cl}: 5.0$ to 6.9 ).

Conclusions: Clinical tests of physical performance are associated with the demand for personal care in older people. Preventive strategies to maintain physical independence may be required in older adults who show a gait speed slower than $1 \mathrm{~m} / \mathrm{s}$. Further research is necessary to confirm these preliminary results.

\section{INTRODUCTION}

Japan is the fastest ageing society on earth and the first large country in the history to have its population start shrinking rapidly from

\section{ARTICLE SUMMARY}

Article focus

- Measures of physical performance may identify older persons with a preclinical stage of disability.

- However, it is unclear which performance test and cut-point are the most useful to screen for risk of functional dependence in older Japanese people.

- The purpose of this study was to identify appropriate clinical tests for determining the risk of functional dependence in older Japanese people.

Key messages

- Clinical tests of physical performance were associated with a functional decline in older people.

- Preventive strategies to avoid personal care may be required in older adults who show a gait speed slower than $1 \mathrm{~m} / \mathrm{s}$.

Strengths and limitations of this study

- Strengths of this study include a large sample size and performance-based assessment, which could determine actual physical capacity and predict subsequent physical disability in older people living in the community.

- We analysed cross-sectional data. Therefore, further investigation of the validity of these tests in predicting the risk of disability in older people using a prospective study design is recommended.

natural causes. The life expectancy of Japanese people (mean age: men, 79.4 years; women, 85.9 years) is at the highest level in the world. The population of Japan, which currently stands at 127 million, is expected to fall to just under 100 million in the next 40 years. By 2050, 4 of 10 adults in Japan will be older than 65 years of age. Japan implemented the national social long-term care insurance (LTCI) system on 1 April 2000. Every Japanese person aged 65 and older is eligible for benefits based strictly on physical and mental frailty or disability. ${ }^{1}$ In June 2006, the Japanese government implemented a major LTCI reform that focused on preventive benefits for the 
population at high risk of disability (ie, physical and/or cognitive frailty), to contain the skyrocketing costs of the LTCI. ${ }^{2}$

Physical frailty increases with advancing age and is a major risk factor for dependency, institutionalisation, and mortality. ${ }^{3-5}$ People with a disability have higher healthcare needs and use compared with those without a disability. ${ }^{6}$ Although the biggest risk factor for future frailty is advancing age, other factors that are possibly modifiable through interventions should not be ignored. For the purpose of targeting risk factors for future frailty, adequate assessment of individual people may be required. One of the main characteristics of the elderly population is its heterogeneity, with elderly people in the same age range showing a wide variance with regard to their risk of disability. To prevent frailty or disability, population-based intervention programmes should be targeted at the population at risk. A feasible and valid screening tool available for research and clinical settings is required to identify target populations. The Interventions on the Frailty Working Group developed recommendations to screen, recruit, evaluate and retain frail older persons in clinical trials. ${ }^{7}$ They reported that most researchers focus on the following domains for identification of physical frailty: mobility, such as lower extremity performance and gait abnormalities; muscle weakness; poor exercise tolerance; unstable balance and factors related to body composition, such as weight loss, malnutrition and muscle loss. ${ }^{7}$

In an effort to select tailored preventive programmes in the Japanese LTCI system, those at high risk for subsequent disability are identified by a basic functional status questionnaire. Although the questionnaire is relatively quick to administer, a performance-based assessment could determine actual physical capacity and more accurately predict subsequent physical disability in communityliving older people. Guralnik et $a l^{\beta}$ reported that measures of physical performance may identify older persons with a preclinical stage of disability who may benefit from interventions to prevent the development of frank disability. A previous study identified that a rapid gait test was more likely than other mobility performance tests to discriminate older women at high risk of frailty based on the Japanese LTCI system. ${ }^{9}$ However, which performance tests including upper and lower limb muscle functions and which cut-points are the most useful to screen for the demand for personal care are not clear. This study investigated the relationships between performance-based physical assessments and demand for personal care in older people using two large sample cohorts in Japan.

\section{METHODS}

\section{Participants}

We performed a national study of 10351 individuals aged 65 years and older who had received personal care $(n=6791)$ and those who had not received personal care $(n=3560)$. The study included individuals who were enrolled in the Obu Study of Health Promotion for the Elderly (OSHPE) and the Tsukui Ordered Useful Care for Health (TOUCH) programme. To enrol in the OSHPE, an individual was recruited from Obu, Japan, which is a residential suburb of Nagoya. Inclusion criteria required that the participant was aged 65 years or older at examination in 2011 or 2012, lived in Obu, and had not participated in another study. Exclusion criteria stipulated that participants be certified as needing support or care by the Japanese public LTCI system, had disability in basic activities of daily living, and could not carry out performance-based assessments. To enrol in the TOUCH programme, an individual had to be 65 years or older and certified as needing support or care from the Japanese public LTCI system. Detailed information was provided in a previous study. ${ }^{10}$ In brief, TOUCH sites (241 day-care centres) are located throughout Japan and provide comprehensive, facilitybased day-care services (eg, bath, lunch, physical and cognitive recreational activities and physical exercise). Most TOUCH clients have some physical disability and frailty, defined as the presence of weakness, low physical activity and/or slow gait speed, in accordance with the widely accepted definition of frailty. ${ }^{7}$

A total of 10351 older participants (mean age, $78.8 \pm 8.0$ years) underwent performance-based assessments. Informed consent was obtained from all participants prior to their inclusion in the study, and the Ethics Committee of the National Centre for Geriatrics and Gerontology approved the study protocol.

\section{Performance-based assessment}

The assessment measures were conducted by welltrained staff who had nursing, physiotherapy, occupational therapy or similar qualifications. Prior to start of the study, all staff received training from the authors in the correct protocols for administering all of the assessment measures. The assessment included several physical tests. Upper and lower limb muscle functions were assessed with the grip strength (GS) and the chair stand test (CST), respectively. ${ }^{11}$ Gait function was assessed with walking time tests conducted at a comfortable pace (comfortable walking speed, CWS) and with the timed up-and-go (TUG) test. ${ }^{12}$

GS was measured in kilograms in the participant's dominant hand using a Smedley-type handheld dynamometer (GRIP-D; Takei Ltd, Niigata, Japan). The CST involved sitting down and standing up five times, using a chair without an armrest. The score was the time taken to complete the task in seconds. Participants were asked to exert their maximum effort in GS and CST. CWS was measured in seconds with a stopwatch. Participants were asked to walk on a flat and straight surface at their CWS. Two markers were used to indicate the start and end of the path, and a $2 \mathrm{~m}$ and over approach was allowed before reaching the start marker so that participants can walk at their comfortable pace within the timed path. They were instructed to continue walking past the end 
of the path for a further $2 \mathrm{~m}$ and over to ensure that the walking pace was consistent throughout the task. The TUG test involved rising from a chair, walking $3 \mathrm{~m}$, turning around, walking back to the chair and sitting down. ${ }^{12}$ The TUG test is one of the most frequently used tests of balance and gait, and is often used to assess fall risk in older people. ${ }^{13}$ The time to complete the TUG test was measured, in seconds, at each participant's usual pace. Both walking tests were measured once, and if a walking aid was nomally used inside the home, this aid was used during the tests.

\section{Cognitive function}

The Mini-Mental State Examination (MMSE) ${ }^{14}$ for the OSHPE population and the Mental Status Questionnaire (MSQ) for individuals enrolled in the TOUCH programme were used to measure cognitive functioning, and were used as potential confounders in the association between performance-based physical assessments and functional dependence. ${ }^{15}$ Individuals with 23 or fewer points on the MMSE and three or more errors on the MSQ were considered to have cognitive impairment. ${ }^{15} 16$

\section{Statistical analysis}

Demographic and clinical variables were compared between the participants with and those without personal care using Student $t$ tests for continuous variables and $\chi^{2}$ tests for categorical variables. To compare the predictive ability of the study measures, receiver-operated characteristic (ROC) curves were inspected to determine cut-points for each test that best discriminated between the individuals with and those without personal care. Cut-points for maximising the sensitivity and specificity for each test were determined using the Youden index. ${ }^{17}$ The area under the curve (AUC), sensitivity and specificity were then calculated for the cut-points. We used multivariate logistic regression analyses to determine ORs and $95 \%$ CIs, and to assess independent associations of the cut-points of physical performance measures for demand for personal care. The participants were divided into two groups according to the cut-point of the performance-based physical assessments. Covariates were added sequentially to the logistic model to evaluate the associations at different levels of adjustment. Model 1 included each performance-based physical assessment, and model 2 included the model 1 variables plus age, sex and cognitive impairment as determined by the MMSE or MSQ. Model 3 included all performance-based physical assessments plus age, sex and cognitive impairment. The participants were then divided into five groups as follows: individuals with no risk and those with 1, 2, 3 or 4 risks, according to the number of risks identified by the cut-points of the performance-based physical assessments. The ORs and 95\% CIs for the number of risks were calculated adjusted for age, sex and cognitive impairment. All statistical contrasts were made at the 0.05 level of significance, and all data management and statistical computations were performed using the
IBM SPSS Statistics V.19.0 software package (SPSS Inc, Chicago, Illinois, USA).

\section{RESULTS}

\section{Comparison between participants with and those without} personal care

Table 1 shows the characteristics of the participants. The participants with personal care were significantly older $(p<0.001)$, included a higher number of women $(p<0.001)$ and a higher number of persons with cognitive impairment $(p<0.001)$ than those without personal care. For the comparison of performance-based assessments, the participants with personal care had significantly lower scores on all physical tests $(\mathrm{p}<0.001)$ compared

Table 1 Characteristics of the participants

\begin{tabular}{|c|c|c|}
\hline & $\begin{array}{l}\text { Participants } \\
\text { with personal } \\
\text { care }(n=6791)\end{array}$ & $\begin{array}{l}\text { Participants } \\
\text { without } \\
\text { personal care } \\
(n=3560)\end{array}$ \\
\hline Age (years)* & $82.6 \pm 6.7$ & $71.8 \pm 5.2$ \\
\hline Sex, women, $n(\%)^{\star}$ & $4720(69.5)$ & $1793(50.4)$ \\
\hline $\begin{array}{l}\text { Cognitive impairments, } \\
\mathrm{n}(\%)^{*}\end{array}$ & $2962(43.6)$ & $562(15.8)[8]$ \\
\hline $\mathrm{GS}(\mathrm{kg})^{*}$ & $16.3 \pm 6.9$ & $27.3 \pm 7.8$ \\
\hline $\operatorname{CST}(s)^{*}$ & $13.0 \pm 5.6$ & $8.6 \pm 2.4$ \\
\hline $\operatorname{CWS}(\mathrm{m} / \mathrm{s})^{*}$ & $0.7 \pm 0.3$ & $1.2 \pm 0.2$ \\
\hline TUG $(s)^{*}$ & $16.6 \pm 7.7$ & $8.9 \pm 1.8$ \\
\hline \multicolumn{3}{|c|}{ Care level in the LTCI, n (\%) } \\
\hline Support need level 1 & $804(11.8)$ & $0(0)$ \\
\hline Support need level 2 & $1112(16.4)$ & $0(0)$ \\
\hline Care need level 1 & 2057 (30.3) & $0(0)$ \\
\hline Care need level 2 & $1687(24.8)$ & $0(0)$ \\
\hline Care need level 3 & $842(12.4)$ & $0(0)$ \\
\hline Care need level 4 & $257(3.8)$ & $0(0)$ \\
\hline Care need level 5 & $32(0.5)$ & $0(0)$ \\
\hline \multicolumn{3}{|c|}{ Disability of basic ADLs, $n(\%)$} \\
\hline Eating & 105 (1.5) [136] & $0(0)$ \\
\hline Grooming & $398(5.9)[136]$ & $0(0)$ \\
\hline Bathing & $\begin{array}{l}1374(20.2) \\
{[136]}\end{array}$ & $0(0)$ \\
\hline Locomotion & $\begin{array}{l}745(11.0) \\
{[136]}\end{array}$ & $0(0)$ \\
\hline Stairs & $\begin{array}{l}1508(22.2) \\
{[136]}\end{array}$ & $0(0)$ \\
\hline
\end{tabular}

Individuals with 23 or fewer points on the MMSE in the participants without personal care and with three or more errors on the MSQ in the participants with personal care are considered to have cognitive impairment. Beneficiaries of the LTCI can use multiple services for which they are eligible, according to their care plan up to the maximum amount ( $£ 382$ for Support Level 1; $£ 800$ for Support Level 2; $£ 1275$ for Care Level 1; 11498 for Care Level 2; £2058 for Care Level 3; £2354 for Care Level 4; £2756 for Care Level 5), in principle, for a $10 \%$ copayment and can use more services than covered as long as they pay all the costs for the services beyond the maximum level (calculated at $£ 1=130$ yen). ${ }^{*}$ Comparison between the participants with and without personal care; $p<0.001$, [ ] missing value.

CST, chair stand test; CWS, comfortable walking speed; GS, grip strength; LTCI, long-term care insurance; MMSE, mini-mental state examination; MSQ, mental status questionnaire; TUG, timed up-and-go test. 


\begin{tabular}{|c|c|c|c|c|}
\hline & Criterion & Sensitivity & Specificity & AUC \\
\hline \multicolumn{5}{|l|}{ GS (kg) } \\
\hline Men & $<26$ & 74 & 89 & 0.88 \\
\hline Women & $<17$ & 80 & 88 & 0.90 \\
\hline \multicolumn{5}{|l|}{$\operatorname{CST}(s)$} \\
\hline Men & $\geq 10$ & 72 & 74 & 0.79 \\
\hline Women & $\geq 10$ & 67 & 77 & 0.78 \\
\hline \multicolumn{5}{|l|}{ CWS (m/s) } \\
\hline Men & $<1.0$ & 86 & 85 & 0.92 \\
\hline Women & $<1.0$ & 90 & 85 & 0.94 \\
\hline \multicolumn{5}{|l|}{ TUG (s) } \\
\hline Men & $\geq 11$ & 76 & 88 & 0.88 \\
\hline Women & $\geq 11$ & 79 & 89 & 0.90 \\
\hline
\end{tabular}

with those in participants without personal care (table 1). The number of participants with and without personal care who used the walking aid during the walking tests were $2593(38.2 \%)$ and $35(1.0 \%)$, respectively.

\section{Cut-points between participants with and those without personal care}

ROC curve analysis results, showing the performance cut-points for each test and associated statistics, are shown in table 2. The Youden index determined the cutpoints for the demand for personal care as follows: GS in men and women was $<26$ and $<17 \mathrm{~kg}$, respectively; CST was $\geq 10 \mathrm{~s}$, CWS was $<1.0 \mathrm{~m} / \mathrm{s}$ and TUG was $\geq 11 \mathrm{~s}$ for both sexes. The CWS score had the highest AUC for discriminating the demand for personal care and displayed good sensitivity and specificity (85-90\%). High AUCs were also found for GS and TUG, as well as fair to good sensitivity and specificity (74-80\%).

\section{Relationships between cut-points and risk of disability}

The multiple logistic regression models showed significant relationships between physical performances and the demand for personal care (table 3). The demand for personal care was most closely related to CWS in model 1 ( $\mathrm{OR}=34.7 ; 95 \%$ CI 30.9 to 39.0$)$. These results remained essentially unchanged after controlling for age, sex, cognitive impairment and other physical performance tests. In the final model (model 3), the highest OR of factors related to the demand for personal care was for CWS (OR=5.9; 95\% CI 5.0 to 6.9 ). Figure 1 shows the distribution of CWS for participants with personal care. Participants who walked $1.1 \mathrm{~m} / \mathrm{s}$ and faster had the lowest amount of personal care $(20 \%)$. The rate of participants with personal care increased rapidly with a CWS slower than $1.1 \mathrm{~m} / \mathrm{s}$, and $90 \%$ of participants with a CWS slower than $0.8 \mathrm{~m} / \mathrm{s}$ had personal care (figure $1 \mathrm{~A}$ ). The rate of functional decline increased rapidly for individuals walking slower than $1 \mathrm{~m} / \mathrm{s}$ in women (figure $1 \mathrm{C}$ ) rather than men (figure 1B), and with the rate of functional decline reaching $90 \%$ when CWS was slower than $0.8 \mathrm{~m} / \mathrm{s}$ in both sexes (figure $1 \mathrm{~B}, \mathrm{C}$ ).

There was a significant relationship between the number of risks based on the physical performance tests and the demand for personal care. The ORs and 95\% CIs for personal care in participants with $1,2,3$ and 4 risks were 3.1 (2.6 to 3.8 ), 10.6 (8.7 to 13.1 ), 35.6 (28.6 to 44.5 ) and 141.3 (103.6 to 192.7), respectively, compared with participants without risks $(\mathrm{p}<0.001)$. Figure 2 shows the distributions of the number of risks for demand for personal care. The rates of participants with personal care who had no risk, 1, 2 and 3 or more risks were $8.7 \%$, $38.5 \%, 75.6 \%$ and $90.0 \%$, respectively (figure 2 ).

\section{DISCUSSION}

Neuromuscular function, including muscle strength, balance and gait, and cognitive function are important risk factors for disability. Performance-based assessment of these factors can be used to identify people at an increased risk of future functional decline. We examined the use of various measures to identify the most useful measure for screening the demand for personal care.

\section{Cut-points of demand for personal care}

In the current study, univariate analyses identified all physical tests as being able to discriminate between participants with and those without personal care. When performance was dichotomised for cut-points, GS, CST, CWS and TUG retained statistically significant relationships with personal care. The CWS test (cut-point, $1 \mathrm{~m} / \mathrm{s}$ ) displayed the highest OR in the final model, with good sensitivity and specificity with respect to

Table 3 Relationships between physical performances and the demand for personal care

\begin{tabular}{lccc}
\hline & Model 1 OR (95\% Cl) & Model 2 OR (95\% Cl) & Model 3 OR (95\% Cl) \\
\hline GS (men: $<26$ vs $\geq 26 \mathrm{~kg}$, women: $<17$ vs $\geq 17 \mathrm{~kg})$ & $20.9(18.6 \text { to } 23.5)^{\star}$ & $8.5(7.4 \text { to } 9.7)^{\star}$ & $4.1(3.5 \text { to } 4.8)^{*}$ \\
CST $(\geq 10$ vs $<10 \mathrm{~s})$ & $6.6(6.1 \text { to } 7.3)^{*}$ & $4.1(3.7 \text { to } 4.7)^{*}$ & $1.3(1.1 \text { to } 1.5)^{*}$ \\
CWS $(<1$ vs $\geq 1 \mathrm{~m} / \mathrm{s})$ & $34.7(30.9 \text { to } 39.0)^{\star}$ & $17.5(15.3 \text { to } 20.0)^{*}$ & $5.9(5.0 \text { to } 6.9)^{*}$ \\
TUG $(\geq 11$ vs $<11 \mathrm{~s})$ & $27.1(24.1 \text { to } 30.5)^{\star}$ & $15.3(13.4 \text { to } 17.6)^{*}$ & $4.0(3.4 \text { to } 4.8)^{*}$ \\
\hline
\end{tabular}

${ }^{*} p<0.01$.

Model 1 was crude ORs and Model 2 was adjusted for age, sex and cognitive impairment. Model 3 was adjusted for age, sex, cognitive impairment and physical performances.

CST, chair stand test; CWS, comfortable walking speed; GS, grip strength; TUG, timed up-and-go test. 
Figure 1 Comfortable walking speed distributions of participants with personal care in all participants (A), men (B) and women $(\mathrm{C})$. The rate of participants with personal care markedly decreased at $1.0 \mathrm{~m} / \mathrm{s}$ and faster at a comfortable walking speed.

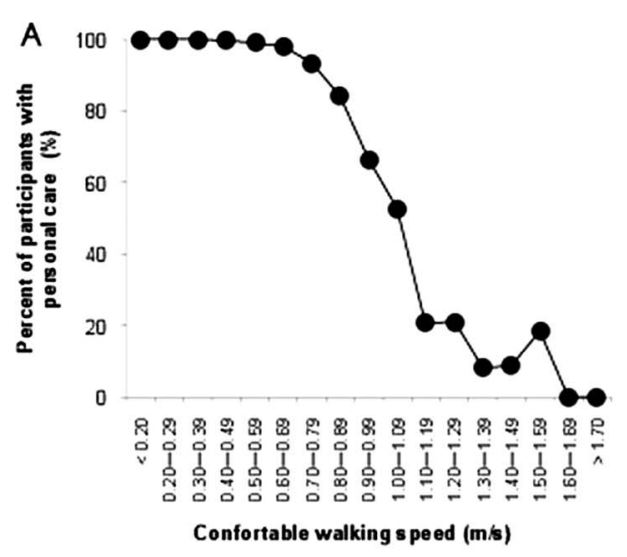

B

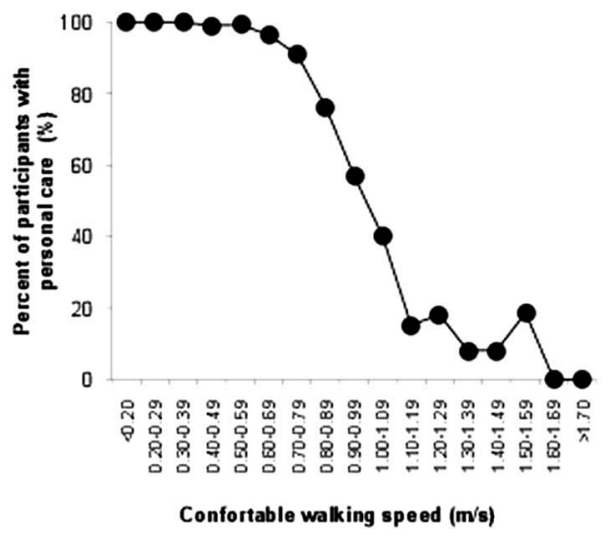

identifying participants with personal care. At identified cut-points, GS (men, $26 \mathrm{~kg}$; women, $17 \mathrm{~kg}$ ), CST (10 s) and TUG (11 s) could also significantly discriminate participants with personal care with sensitivities and specificities of $67-89 \%$. This result highlights what can occur when dichotomised rather than continuous data are used. There is an associated loss of information and

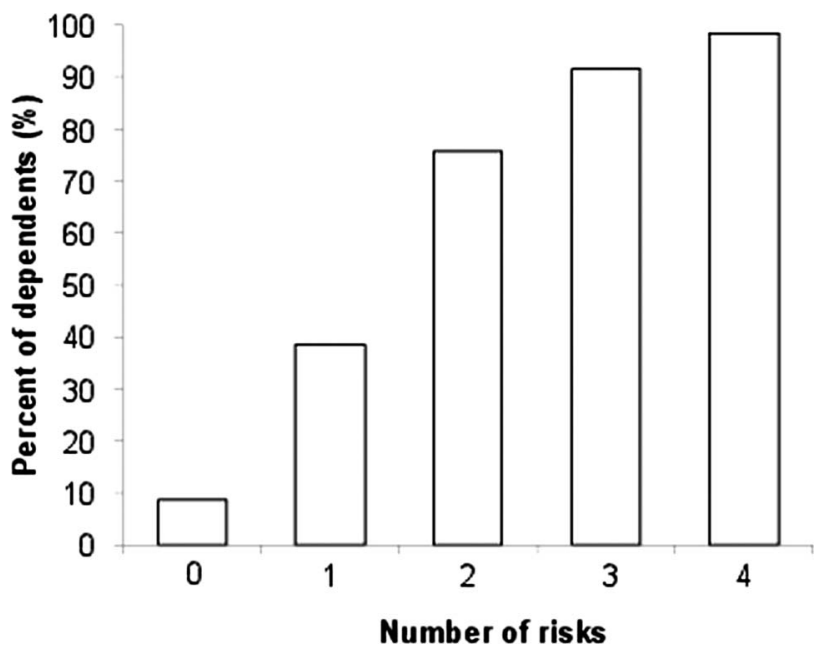

Figure 2 Participants with personal care according to the number of risks identified by cut-points of physical performance tests. Percentages of participants with personal care who had no risk, 1,2 and 3 or more risks were $8.7 \%$, $38.5 \%, 75.6 \%$ and $90 \%$, respectively. reduced predictive accuracy as a trade-off for ease of scoring and test interpretation. These results, however, are consistent with previous findings that showed associations between measures of muscle strength and mobility and functional decline. ${ }^{18}$

\section{Gait speed and personal care}

Gait velocity, as measured by the CWS test in this study, has been consistently reported to differentiate between participants with and those without personal care, with frail older persons walking significantly slower, ${ }^{10} 19$ and has proved to be a strong predictor of adverse events, such as disability, ${ }^{18}$ 20-25 mortality, ${ }^{21} 222627$ hospitalisation $^{21222428}$ and falls. $^{28}{ }^{29}$ Gait slowing, which occurs in the latest stages of life, suggests that mobility is so central to life that energy is shifted away from walking activity only when other vital activities are threatened, ${ }^{30}$ which may lead to increased functional independence. In addition, a slower walking speed is an associated factor for subsequent dementia. ${ }^{31}$ Dementia is one of the most important factors of health problems for functional decline in the aged population. For our study sample, the cut-point for CWS was $1 \mathrm{~m} / \mathrm{s}$, which is the critical point for future functional decline in community-dwelling older people determined by previous studies. $^{18} \quad 21 \quad 22 \quad 24 \quad 25$ These results suggest that walking speed may be the most crucial measurement to determine the demand for personal care in older adults. Measurement of walking speed is reliable, valid, 
sensitive, inexpensive, safe, quick and a simple tool. Therefore, measurement of walking speed is suitable to use in community settings as a screening tool and evaluation for the effect of a care prevention programme.

\section{Muscle strength and mobility and personal care}

In the current study, higher ORs were found for GS and TUG, as well as CWS. Hand GS is an estimate of isometric strength in the upper extremity, but also correlates with strength in other muscle groups, ${ }^{32}$ and therefore, is considered an estimate of the overall strength. In addition, GS has proved to be a strong predictor of physical functioning and disability, ${ }^{33}$ morbidity $^{35}$ and mortality. ${ }^{36} 37$ Our findings support previous evidence and add cut-points of $<26 \mathrm{~kg}$ in men and $<17 \mathrm{~kg}$ in women that discriminate those at high-risk for disability in community-living older people. The TUG has been recommended as a screening tool for identifying older people who are at risk for falling. ${ }^{38} 39$ Bischoff et at ${ }^{40}$ proposed a normative cut-point of $12 \mathrm{~s}$ for community-dwelling elderly people between 65 and 85 years of age. In daily clinical practice, elderly persons who perform the TUG in $>12 \mathrm{~s}$ should receive early evaluation and intervention. Our results regarding TUG cut-points are in line with these previous studies.

\section{Strengths and limitations}

Strengths of the present study include a large sample size and we used performance-based assessment, which could determine actual physical capacity and predict subsequent physical disability in community-living older people. However, the present study has a number of limitations. One of the limitations is that we analysed crosssectional data. Therefore, further investigation of the validity of these tests in predicting the risk of disability in older people using a prospective study design is recommended. Another limitation is that many frail older people using healthcare services cannot walk because they have multiple diseases or geriatric syndromes. Non-ambulatory participants were excluded from our study. Therefore, we acknowledge that the study findings may not be generalised to this frailer group.

\section{CONCLUSIONS}

This study provides preliminary evidence that clinical tests of physical performances can predict the risk of disability in older people. Logistic regression analysis selected CWS as the best independent correlate of disability, with good sensitivity and specificity. Further investigation is required, and future research should include a prospective measurement of the risk of disability to more accurately determine the validity of screening tests for this population.

\footnotetext{
Author affiliations

${ }^{1}$ Section of Health Promotion, Department of Support for Independence, Center for Gerontology and Social Science, National Center for Geriatrics and Gerontology, Obu, Aichi, Japan

${ }^{2}$ Research Institute, National Center for Geriatrics and Gerontology, Obu, Aichi, Japan
}

${ }^{3}$ Department of Rehabilitation, Course of Physical Therapy, University of Human Arts and Science, Saitama, Japan

${ }^{4}$ Section of Physical Functioning Activation, Department of Functioning Activation, Center for Gerontology and Social Science, National Center for Geriatrics and Gerontology, Obu, Aichi, Japan

Acknowledgements We thank the Obu City Office for the help provided with participant recruitment, and staff at the Tsukui Corporation for their assistance with data collection.

Contributors HS designed and organised the study, analysed and interpreted the data, and drafted the manuscript. TS and MS made substantial contributions to the conception, design, analysis and interpretation of the data, and critically revised the draft. All authors took responsibility for the accuracy and integrity of the study. All authors gave the final approval of the version to be published.

Funding This work received financial support from a grant from the Japanese Ministry of Health, Labour and Welfare (Project for optimising long-term care; B-3) and a grant from the National Center for Geriatrics and Gerontology (Research Funding for Longevity Sciences; 22-16). The funding source played no role in the design or conduct of the study; collection, management, analysis or interpretation of the data; or preparation, review or approval of the manuscript.

\section{Competing interests None.}

Patient consent Obtained.

Ethics approval Ethics Committee of the National Center for Gerontology and Geriatrics.

Provenance and peer review Not commissioned; externally peer reviewed.

Data sharing statement No additional data are available.

\section{REFERENCES}

1. Tsutsui T, Muramatsu N. Care-needs certification in the long-term care insurance system of Japan. J Am Geriatr Soc 2005;53:522-7.

2. Tsutsui T, Muramatsu N. Japan's universal long-term care system reform of 2005: containing costs and realizing a vision. J Am Geriatr Soc 2007;55:1458-63.

3. Fried LP, Ettinger WH, Lind B, et al. Physical disability in older adults: a physiological approach. Cardiovascular Health Study Research Group. J Clin Epidemiol 1994;47:747-60.

4. Ensrud KE, Ewing SK, Taylor BC, et al. Frailty and risk of falls, fracture, and mortality in older women: the study of osteoporotic fractures. J Gerontol A Biol Sci Med Sci 2007;62:744-51.

5. Woods NF, LaCroix AZ, Gray SL, et al. Frailty: emergence and consequences in women aged 65 and older in the Women's Health Initiative Observational Study. J Am Geriatr Soc 2005;53:1321-30.

6. Guralnik JM, Fried LP, Simonsick EM, et al. The women's health and aging study: health and social characteristics of older women with disability. Bethesda, MD: National Institute of Aging, NIH Pub. No: 95-4009, 1995.

7. Ferrucci L, Guralnik JM, Studenski S, et al. Designing randomized, controlled trials aimed at preventing or delaying functional decline and disability in frail, older persons: a consensus report. $J \mathrm{Am}$ Geriatr Soc 2004;52:625-34.

8. Guralnik JM, Ferrucci L, Simonsick EM, et al. Lower-extremity function in persons over the age of 70 years as a predictor of subsequent disability. N Engl J Med 1995;332:556-61.

9. Kim MJ, Yabushita N, Kim MK, et al. Mobility performance tests for discriminating high risk of frailty in community-dwelling older women. Arch Gerontol Geriatr 2010;51:192-8.

10. Shimada H, Suzukawa M, Tiedemann A, et al. Which neuromuscular or cognitive test is the optimal screening tool to predict falls in frail community-dwelling older people? Gerontology 2009;55:532-8.

11. Csuka M, McCarty DJ. Simple method for measurement of lower extremity muscle strength. Am J Med 1985;78:77-81.

12. Podsiadlo D, Richardson S. The timed 'Up \& Go': a test of basic functional mobility for frail elderly persons. $J$ Am Geriatr Soc 1991;39:142-8.

13. National Institute for Clinical Excellence. Clinical practice guideline for the assessment and prevention of falls in older people. London: Royal College of Nursing, 2004. 
14. Folstein MF, Folstein SE, McHugh PR. 'Mini-mental state'. A practical method for grading the cognitive state of patients for the clinician. J Psychiatr Res 1975;12:189-98.

15. Kahn R, Goldfarb A, Pollack M, et al. Brief objective measures for the determination of mental status in the aged. Am J Psychiatry 1960;117:326-8.

16. Anthony JC, LeResche L, Niaz U, et al. Limits of the 'Mini-Mental State' as a screening test for dementia and delirium among hospital patients. Psychol Med 1982;12:397-408.

17. Perkins NJ, Schisterman EF. The inconsistency of 'optimal' cutpoints obtained using two criteria based on the receiver operating characteristic curve. Am J Epidemiol 2006;163:670-5.

18. Shinkai $S$, Watanabe $S$, Kumagai $S$, et al. Walking speed as a good predictor for the onset of functional dependence in a Japanese rural community population. Age Ageing 2000;29:441-6.

19. Tiedemann A, Shimada $H$, Sherrington $C$, et al. The comparative ability of eight functional mobility tests for predicting falls in community-dwelling older people. Age Ageing 2008;37:430-5

20. Guralnik JM, Ferrucci L, Pieper CF, et al. Lower extremity function and subsequent disability: consistency across studies, predictive models, and value of gait speed alone compared with the short physical performance battery. J Gerontol A Biol Sci Med Sci 2000;55:M221-31.

21. Cesari M, Kritchevsky SB, Penninx BW, et al. Prognostic value of usual gait speed in well-functioning older people-results from the Health, Aging and Body Composition Study. J Am Geriatr Soc 2005;53:1675-80.

22. Cesari M, Kritchevsky SB, Newman AB, et al. Added value of physical performance measures in predicting adverse health-related events: results from the Health, Aging and Body Composition Study. J Am Geriatr Soc 2009;57:251-9.

23. Onder G, Penninx BW, Ferrucci L, et al. Measures of physical performance and risk for progressive and catastrophic disability: results from the Women's Health and Aging Study. J Gerontol A Biol Sci Med Sci 2005;60:74-9.

24. Studenski S, Perera S, Wallace D, et al. Physical performance measures in the clinical setting. J Am Geriatr Soc 2003; $51: 314-22$.

25. Simonsick EM, Newman AB, Visser M, et al. Mobility limitation in self-described well-functioning older adults: importance of endurance walk testing. J Gerontol A Biol Sci Med Sci 2008;63:841-7.

26. Markides KS, Black SA, Ostir GV, et al. Lower body function and mortality in Mexican American elderly people. J Gerontol A Biol Sci Med Sci 2001;56:M243-7.
27. Studenski S, Perera S, Patel K, et al. Gait speed and survival in older adults. JAMA 2011;305:50-8.

28. Montero-Odasso M, Schapira M, Soriano ER, et al. Gait velocity as a single predictor of adverse events in healthy seniors aged 75 years and older. J Gerontol A Biol Sci Med Sci 2005;60:1304-9.

29. Dargent-Molina $\mathrm{P}$, Favier $\mathrm{F}$, Grandjean $\mathrm{H}$, et al. Fall-related factors and risk of hip fracture: the EPIDOS prospective study. Lancet 1996;348:145-9.

30. Wang L, Larson EB, Bowen JD, et al. Performance-based physical function and future dementia in older people. Arch Intern Med 2006;166:1115-20.

31. Beauchet $\mathrm{O}$, Kressig RW, Najafi B, et al. Age-related decline of gait control under a dual-task condition. J Am Geriatr Soc 2003:51:1187-8.

32. Rantanen T, Era $\mathrm{P}$, Kauppinen $\mathrm{M}$, et al. Maximal isometric muscle strength and socio-economic status, health and physical activity in 75-year-old persons. Aging Phys Act 1994;2:206-20.

33. Ishizaki T, Watanabe S, Suzuki T, et al. Predictors for functional decline among non-disabled older Japanese living in a community during a 3-year follow-up. J Am Geriatr Soc 2000;48:1424-9.

34. Rantanen T, Guralnik JM, Sakari-Rantala R, et al. Disability, physical activity, and muscle strength in older women: the Women's Health and Aging Study. Arch Phys Med Rehabil 1999;80:130-5.

35. Rantanen T, Masaki K, Foley D, et al. Grip strength changes over $27 \mathrm{yr}$ in Japanese-American men. $J$ Appl Physiol 1998;85:2047-53.

36. Rantanen T, Harris T, Leveille SG, et al. Muscle strength and body mass index as long-term predictors of mortality in initially healthy men. J Gerontol A Biol Sci Med Sci 2000;55:M168-73.

37. Fujita $Y$, Nakamura $Y$, Hiraoka J, et al. Physical-strength tests and mortality among visitors to health-promotion centers in Japan. J Clin Epidemiol 1995;48:1349-59.

38. Sletvold O, Tilvis R, Jonsson A, et al. Geriatric work-up in the Nordic countries. The Nordic approach to comprehensive geriatric assessment. Dan Med Bull 1996;43:350-9.

39. Okumiya K, Matsubayashi K, Nakamura T, et al. The timed 'up \& go' test is a useful predictor of falls in community-dwelling older people. $J$ Am Geriatr Soc 1998;46:928-30.

40. Bischoff HA, Stahelin HB, Monsch AU, et al. Identifying a cut-off point for normal mobility: a comparison of the timed 'up and go' test in community-dwelling and institutionalised elderly women. Age Ageing 2003;32:315-20. 